\title{
Nonlinear repolarization in optical fibers: polarization attraction with copropagating beams
}

\author{
Victor V. Kozlov, ${ }^{1,2, *}$ Konstantin Turitsyn, ${ }^{3}$ and Stefan Wabnitz ${ }^{1}$ \\ ${ }^{1}$ Dipartimento di Ingegneria dell' Informazione, Università di Brescia, Via Branze 38, 25123 Brescia, Italy \\ ${ }^{2}$ Department of Physics, St. Petersburg State University, Petrodvoretz, St. Petersburg 198504, Russia \\ ${ }^{3}$ Department of Mechanical Engineering, Massachusetts Institute of Technology, Cambridge, Massachusetts 02139, USA \\ ${ }^{*}$ Corresponding author: victor.kozlov@ing.unibs.it
}

Received July 19, 2011; revised September 12, 2011; accepted September 14, 2011;

posted September 19, 2011 (Doc. ID 151321); published October 12, 2011

\begin{abstract}
We propose a type of lossless nonlinear polarizer, novel to our knowledge, a device that transforms any input state of polarization (SOP) of a signal beam into one and the same well-defined SOP toward the output, and perform this without any polarization-dependent losses. At the polarizer output end, the signal SOP appears to be locked to the input pump SOP. The polarizer is based on the nonlinear Kerr interaction of copropagating signal and pump beams in a telecom or randomly birefringent optical fiber. (c) 2011 Optical Society of America

OCIS codes: $\quad 060.4370,230.5440,230.1150,230.4320$.
\end{abstract}

Over the past years there has been a growing interest in the all-optical control of the state of polarization (SOP) of a light beam. Particular attention was paid to the development of nonlinear polarizers, a class of devices that allows for the intelligent transformation of an arbitrary input SOP of a signal beam into one and the same well-defined SOP toward the output. This output SOP is imposed by the SOP of the pump beam, thereby implementing all-optical control over light polarization. We are interested here in nonlinear lossless polarizers (NLPs): these devices utilize a conservative type of nonlinearity, e.g., the Kerr nonlinearity. Unlike conventional polarizers, the NLPs fully preserve the energy of the signal beam and most importantly are free of polarizationdependent losses.

The first type of NLP was based on photorefractive two-beam coupling, and it was experimentally demonstrated in [1]. The seconds to minutes response time of nonlinearity in photorefractive materials prevents their use in high-speed data processing devices. From this viewpoint, NLPs based on the virtually instantaneous Kerr nonlinearity of optical fibers appear more suitable for telecom application needs. The experimental study of such devices was pioneered in Dijon: first results were reported in [2] and followed by [3,4]. Indeed, the NLP proposed in [프 was able to smooth at its output microsecond-range polarization bursts of the input signal beam. As theoretically and experimentally shown in [5], the microsecond response range is the practical limit for NLPs using telecom fibers. As such, one is not capable of fully exploiting the instantaneous nature of Kerr nonlinearity. The reason is that these NLPs operate in the counterpropagating geometry. Here we propose and validate an NLP based on the interaction of copropagating beams, which is therefore free from the above limitation. Its response time is limited only by the electronic response time of the silica (about few femtoseconds). This NLP is capable of repolarizing even unpolarized incoherent light, such as amplified spontaneous emission, providing that its polarization fluctuations are slower than the Kerr response time of the fiber.

In this Letter we are going to deal with a minimal model. Namely, we consider two intense CW beams copropagating along the $z$ axis through a lossless fiber of total length $L$. The randomness of the fiber birefringence is fully taken into account; see [6,7] for details. The evolution equations for the Stokes vectors of the signal and pump beams, $\mathbf{S}^{(s)}=\left(S_{1}^{(s)}, S_{2}^{(s)}, S_{3}^{(s)}\right)^{T}$ and $\mathbf{S}^{(p)}=$ $\left(S_{1}^{(p)}, S_{2}^{(p)}, S_{3}^{(p)}\right)^{T}$, are

$$
\begin{aligned}
& d_{z} \mathbf{S}^{(s)}=\gamma \mathbf{S}^{(s)} \times \mathbf{J}_{x}(\boldsymbol{z}) \mathbf{S}^{(p)}, \\
& d_{z} \mathbf{S}^{(p)}=\gamma \mathbf{S}^{(p)} \times \mathbf{J}_{x}(z) \mathbf{S}^{(s)},
\end{aligned}
$$

where $d_{z}$ means derivative with respect to $z, \gamma$ is the Kerr coefficient, $\times$ the notation for cross product, and $\mathbf{J}_{x}(\boldsymbol{z})=$ $\operatorname{diag}\left(J_{x 1}(z), J_{x 2}(z), J_{x 3}(z)\right)$ is the cross-polarization tensor, whose $z$-varying diagonal components can be found from a set of linear ordinary differential equations with constant coefficients as derived in [ㅈ,]. The coefficients in the previous equations depend on the magnitudes of the birefringence $\Delta \beta\left(\omega_{s, p}\right)$ at the signal $\omega_{s}$ and pump $\omega_{p}$ carrier frequencies, as well as on the birefringence correlation length $L_{c}$. The randomly birefringent fiber is characterized by its polarization mode dispersion (PMD) coefficient $D_{p}=2 \sqrt{2} \pi \sqrt{L_{c}} /\left[L_{B}\left(\omega_{s}\right) \omega_{s}\right]$, where $L_{B}\left(\omega_{s}\right)=\pi / \Delta \beta\left(\omega_{s}\right)$ is the beat length at $\omega_{s}$. Another important length scale in the problem is the PMD diffusion length $L_{d}$, which is defined as $L_{d}^{-1}=\frac{1}{3} D_{p}^{2}\left(\omega_{p}-\omega_{s}\right)^{2}$. Equations (1) and (2) are strictly valid in two limits: $L$, $L_{\mathrm{NL}} \ll \bar{L}_{d}$, which we call here the Manakov limit, and $L, L_{\mathrm{NL}} \gg L_{d}$ which is named the diffusion limit. As $\mathbf{J}_{x}(\boldsymbol{z})=\operatorname{diag}(0,0,0)$ in the diffusion limit, such a case is not interesting to us because the polarization mixing, which is vitally important for the operation of the NLP, is absent. In the Manakov limit, in which we restore the vector Manakov equation without group-velocity dispersion (GVD) and walk-off terms, we get $\mathbf{J}_{x}=-\frac{8}{9} \operatorname{diag}(1,1,1)$. In this case the NLP operates most efficiently; therefore, this limit is of primary interest to us.

As in counterpropagating NLPs [4, $, 5,7]$, we fix $\gamma=$ $1(\mathrm{Wkm})^{-1}$, the pump wavelength $\lambda_{p}=1.55 \mu \mathrm{m}$, and the signal power $S_{0}^{(s)}=1 \mathrm{~W}$. We apply a fully polarized pump 
beam at the fiber input end along with a polarization scrambled signal beam. Our simulations show that, in agreement with the symmetry of Eqs. (1) and (2), the comparison of results generated for different pump SOPs reveals no visible difference. For definiteness, we fix the pump SOP at $(1,0,0)$. The scrambled signal beam is composed of a set of $N>1000$ beams with SOPs uniformly distributed over the entire Poincaré sphere, so that the corresponding degree of polarization $(\mathrm{DOP})=$ $\sqrt{\sum_{i=1}^{3}\left\langle S_{i}^{(s)}\right\rangle^{2}} / S_{0}^{(s)}$ is zero (here the average is taken over all $N$ realizations of the scrambled beams). The model gives a statistically identical behavior when an unpolarized signal beam is applied at the input end instead of the set scrambled beams; see [5].

In the numerical experiments based on Eqs. (1) and (2) whose results are shown in Fig. 1, we vary the pump beam power, $S_{0}^{(p)}$ and follow the DŌP of the signal beam at the output end of a $2.2 \mathrm{~km}$ long fiber as a function of the PMD diffusion length $L_{d}$. Our goal is to repolarize the signal beam as strongly as possible so that the signal DOP reaches a value that is close to unity. As can be seen in Fig. 1, the largest possible DOP of this setup is 0.73 . Such a beam is polarized only partially.

Whenever a better DOP is required, two or three polarizers can be cascaded; see Fig. 2(a). At each successive stage the repolarized beam exiting the previous polarizer experiences further repolarization, which results in an overall improved DOP. As seen in Fig. 2(b), three cascaded NLPs allow us to reach DOP as high as 0.98 .

A polarizer transforms an arbitrary input signal SOP into a well-defined SOP. In our case, the repolarization process is an intelligent lossless attraction/pulling toward a particular SOP. The signal SOP tends to align not with the local value of the pump SOP, as it occurs with Raman polarizers, [6] , and not with the SOP derived in a special

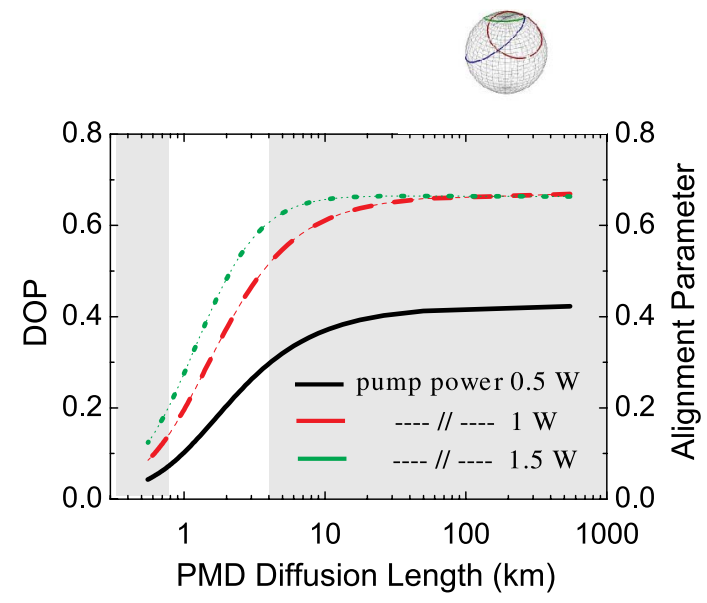

Fig. 1. (Color online) DOP (thick lines) and alignment parameter (thin lines) of the signal beam as a function of the PMD diffusion length for three different values of the pump power: $0.5 \mathrm{~W}$ (black solid), $1 \mathrm{~W}$ (red dashed), $1.5 \mathrm{~W}$ (green dotted). Parameters are $L=2.2 \mathrm{~km}, L_{B}=4 \mathrm{~m}$, channel separation $\Delta \lambda=2 \pi c\left(\omega_{p}-\omega_{s}\right) / \omega_{s}^{2}=5 \mathrm{~nm}$. Shaded regions (left, diffusion limit; right, Manakov limit) indicate the range of validity of the model Eqs. (1) and (2). Above the graph are three representative trajectories on the Poincaré sphere for the case when the pump SOP is along the north pole; the bias of the trajectories toward the north pole is evident.
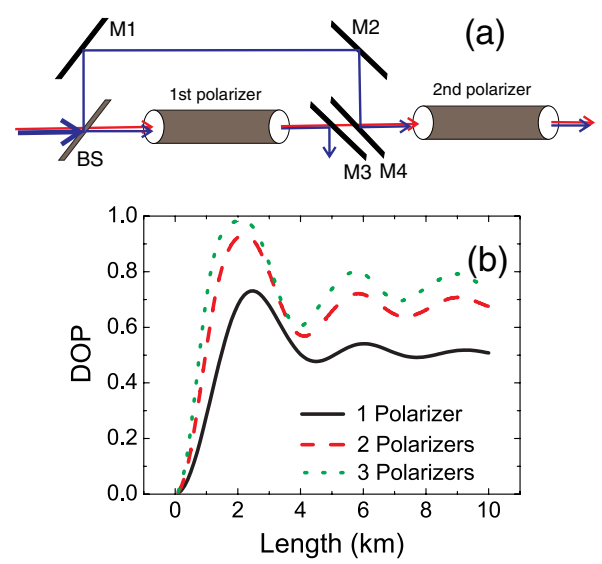

Fig. 2. (Color online) (a) Cascaded scheme with two sequential polarizers. The pump beam (blue line) is split by a $50 / 50$ beam splitter (BS) into two beams, one of which feeds the first polarizer, while the second beam feeds the second polarizer (M1, M2, M3, M4, mirrors). The signal beam (red line) goes through both polarizers without being affected by the BS and mirrors M3 and M4. (b) DOP of the signal beam as a function of the fiber length of a single polarizer plotted using Eq. (5) for three configurations: one polarizer (black solid), two/three sequential identical polarizers (red dashed/green dotted).

manner from the input pump SOP as described in [므, 8 , but it experiences attraction directly to the input pump SOP. Therefore, the proper alignment parameter is provided by the average of the cosine of the angle between the Stokes vectors $\mathbf{S}^{(s)}(L)$ and $\mathbf{S}^{(p)}(0): A_{0 L}=$ $\sum_{i=1}^{3}\left\langle S_{i}^{(s)}(L)\right\rangle S_{i}^{(p)}(0) /\left[S_{0}^{(s)}(L) S_{0}^{(p)}(0)\right]$. The evolution of $A_{0 L}$ as a function of $L_{d}$, which is shown in Fig. 1, fully overlaps with the DOP curves. This confirms that the beam repolarization is closely associated with its alignment to the input pump polarization.

Large values of the PMD diffusion length $L_{d}$ imply either low PMD coefficients or small channel separations. As shown in Fig. 1, a large $L_{d}$ favors the attraction process, i.e., yields the largest DOPs. This confirms that a codirectional NLP performs better when operating in the Manakov limit. Practically speaking, associated physical values of the PMD coefficient are not that small. Indeed, for a channel separation of the order of $1 \mathrm{~nm}$, we find from the condition $L \sim L_{d}$ that the upper bound on the PMD coefficient, $\left(D_{p}\right)_{\mathrm{cr}}$, can be as high as $2 \mathrm{ps} /$ $\sqrt{L(\mathrm{~km})}$. For $L=2.2 \mathrm{~km},\left(D_{p}\right)_{\mathrm{cr}}=1.3 \mathrm{ps} / \sqrt{\mathrm{km}}$.

When considered in the Manakov limit, the governing Eqs. (1) and (2) can be fully solved in analytical form. The equations become

$$
d_{z} \mathbf{S}^{(s)}=-\bar{\gamma} \mathbf{S}^{(s)} \times \mathbf{S}^{(p)}, \quad d_{z} \mathbf{S}^{(p)}=-\bar{\gamma} \mathbf{S}^{(p)} \times \mathbf{S}^{(s)},
$$

with $\bar{\gamma}=\frac{8}{9} \gamma$. Equations (3) lead to three conservation laws: $d_{z}\left[\mathbf{S}^{(s)}+\mathbf{S}^{(p)}\right]=0, d_{z}\left[\mathbf{S}^{(s)} \cdot \mathbf{S}^{(s)}\right]=0$, and $d_{z}\left[\mathbf{S}^{(p)}\right.$. $\left.\mathbf{S}^{(p)}\right]=0$. Here the dot stands for the scalar product. Using the first conservation law and the properties of the cross product $\mathbf{S}^{(p)} \times \mathbf{S}^{(p)}=0$ and $\mathbf{S}^{(s)} \times \mathbf{S}^{(p)}=-\mathbf{S}^{(p)} \times \mathbf{S}^{(s)}$, we may rewrite the equation for $\mathbf{S}^{(s)}$ as

$$
d_{z} \mathbf{S}^{(s)}=\bar{\gamma} \mathbf{\Omega} \times \mathbf{S}^{(s)},
$$



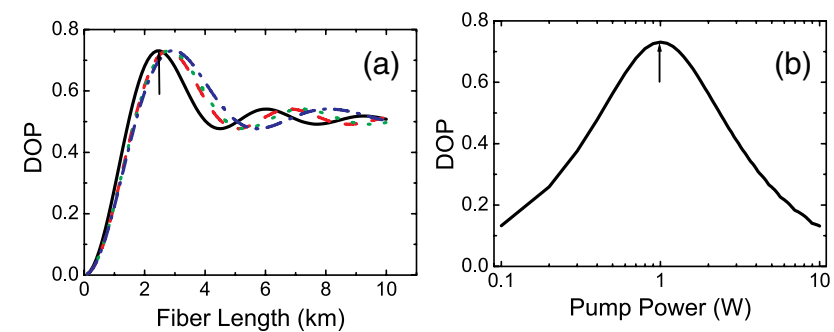

Fig. 3. (Color online) (a) DOP of the signal beam as a function of fiber length for $S_{0}^{(s)}=S_{0}^{(p)}=1 \mathrm{~W}$ : the curve was generated from the analytical Eq. (5) valid in the Manakov limit (black solid), numerical simulation of Eqs. (1) and (2) for $L_{d}=548 \mathrm{~km}$ (red dashed), $L_{d}=55 \mathrm{~km}$ (green dotted), and $L_{d}=21 \mathrm{~km}$ (blue dotted-dashed). (b) Maximal DOP of the signal beam as a function of pump power. For each point on the plot, the maximal DOP was found using Eq. (5) from a set of DOPs obtained for a discrete set of fiber lengths scanned through the interval $[0.1 ; 10] L_{\mathrm{NL}}$. Arrows on plots (a) and (b) indicate the same point in the parameter space.

which describes the precession of the Stokes vector $\mathbf{S}^{(s)}$ around the constant and known vector $\Omega \equiv$ $\left[\mathbf{S}^{(s)}(0)+\mathbf{S}^{(p)}(0)\right]$. The solution of Eq. (q) is well known:

$$
\mathbf{S}^{(s)}(\boldsymbol{z})=\mathbf{b}_{1}+\mathbf{b}_{2} \cos (\Omega \bar{\gamma} \boldsymbol{z})+\mathbf{b}_{3} \sin (\Omega \bar{\gamma} \boldsymbol{z}),
$$

with $\quad \mathbf{b}_{1}=\left(\boldsymbol{\Omega} \cdot \mathbf{S}^{(s)}(0) / \Omega^{2}\right) \boldsymbol{\Omega}, \quad \mathbf{b}_{2}=\mathbf{S}^{(s)}(0)-\mathbf{b}_{1}, \quad \mathbf{b}_{3}=$ $\left(\Omega \times \mathbf{S}^{(s)}(0)\right) / \Omega$. We used this solution to generate Fig. 3 as an example of an ideal (i.e., operating in the Manakov limit) codirectional NLP. Figure 3 suggests that the best performance of the polarizer is reached for equal pump and signal powers, and the fiber length $L \approx 2.2 L_{\mathrm{NL}}\left[L_{\mathrm{NL}}=\right.$ $\left(\bar{\gamma} S_{0}^{(s)}\right)^{-1}$ is the nonlinear length].

Strictly speaking, our model was derived for CW beams since we neglected pulse walk-off terms (owing to the difference of group velocities of pulses in neighboring channels) and GVD terms. However, as long as the length of the fiber is kept shorter than a few kilometers, our simple model gives adequate predictions even for pulses in the picosecond range. For example, when considering the processing of $25 \mathrm{ps}$ pulses, the walk-off distance along $L=2.2 \mathrm{~km}$ is around $2 \mathrm{ps}$ for a channel separation of $1 \mathrm{~nm}$ and with the GVD $\beta_{2}=1 \mathrm{ps} / \mathrm{nm} \cdot \mathrm{km}$. Dispersive pulse broadening is also negligible over the distance $L$, since the dispersion length is $500 \mathrm{~km}$. Thus, our model is readily applicable to telecom processing applications.

The fact that a single polarizer cannot produce a light beam with DOP better than 0.73 can be explained by the bias of the dynamical system trajectories toward the orientation of the initial pump direction on the Poincaré sphere; see above the graph in Fig. 1. More formally, each of the trajectories is a circle centered around the direction of $\Omega$. Assuming that the vector $S^{(p)}(0)$ points to the direction of the north pole, the signal SOP spends more time in the upper hemisphere. However, the signal is not attracted dynamically to the direction of the pump as it is in the case in the counterpropagating configuration.

In conclusion, we proposed an NLP based on the codirectional interaction of two intense beams in a randomly birefringent optical fiber. We have validated the performance of this device by showing that any input SOP of the signal beam is on average attracted to the input SOP of the pump beam toward the device output. Practical issues were also discussed.

The authors appreciate numerous enlightening discussions with A. Aceves. This work was carried out with support from the Programma di Ricerca Scientifica di Rilevante Interesse Nazionale (PRIN2008) project "Nonlinear cross-polarization interactions in photonic devices and systems" and the Cariplo Foundation through the University of Brescia-Massachusetts Institute of Technology/Mechanical Engineering faculty exchange programme.

\section{References}

1. J. E. Heebner, R. S. Bennink, R. W. Boyd, and R. A. Fisher, Opt. Lett. 25, 257 (2000).

2. S. Pitois, G. Millot, and S. Wabnitz, J. Opt. Soc. Am. B 18, 432 (2001).

3. S. Pitois, J. Fatome, and G. Millot, Opt. Express 16, 6646 (2008).

4. J. Fatome, S. Pitois, P. Morin, and G. Millot, Opt. Express 18, $15311(2010)$.

5. V. V. Kozlov, J. Fatome, P. Morin, S. Pitois, G. Millot, and S. Wabnitz, J. Opt. Soc. Am. B 28, 1782 (2011).

6. V. V. Kozlov, J. Nuño, J. D. Ania-Castañón, and S. Wabnitz, Opt. Lett. 35, 3970 (2010).

7. V. V. Kozlov, J. Nuño, and S. Wabnitz, J. Opt. Soc. Am. B 28, 100 (2011).

8. E. Assemat, D. Dargent, A. Picozzi, H. R. Jauslin, and D. Sugny, Opt. Lett. 36, 4038 (2011). 